\title{
Red raspberries can improve motor function in aged rats
}

\author{
Rachel L. Galli ${ }^{\mathrm{a}}$, Amanda N. Carey ${ }^{\mathrm{a}}$, Katharine A. Luskin ${ }^{\mathrm{b}}$, Donna F. Bielinski ${ }^{\mathrm{b}}$ \\ and Barbara Shukitt-Hale ${ }^{\mathrm{b}, *}$ \\ ${ }^{a}$ Department of Psychology, Simmons College, Boston, MA, USA \\ ${ }^{\mathrm{b}}$ USDA-ARS, Human Nutrition Research Center on Aging at Tufts University, Boston, MA, USA
}

Received 21 December 2015; accepted 10 January 2016

\begin{abstract}
.
BACKGROUND: Many foods rich in antioxidant and anti-inflammatory compounds have been shown to increase health and reduce markers of aging. A number of berry fruits high in polyphenols are known to ameliorate age-related declines in cellular, cognitive and behavioral function in rats.

OBJECTIVES: This study evaluated the effectiveness of a red raspberry-supplemented diet on age-sensitive measures of learning, memory and motor performance in aged (19 mo) F344 rats.

METHODS: Red raspberry extract was prepared from fresh whole fruit and incorporated in standard rodent chow to create a $2 \%$ diet. Following ten weeks on a control or $2 \%$ raspberry diet, cognitive and motor performance was assessed using the Morris Water Maze (MWM) and a battery of five psychomotor tasks.

RESULTS: The supplemented diet significantly improved performance on three of the five motor tasks, but did not affect MWM performance. Specifically, old rats fed the $2 \%$ raspberry diet had significantly better performance on the rod and plank walks, which measure psychomotor coordination and balance, and on the inclined screen, which measures muscle tone, strength, stamina and balance.

CONCLUSIONS: Given that falls are the number one health hazard for otherwise healthy older adults, these results may have important implications for increasing healthy aging.
\end{abstract}

Keywords: Red raspberry, polyphenols, ellagitannins, antioxidant, anti-inflammatory, aging, motor performance

\section{Introduction}

Brain-based changes in functioning associated with age are widely accepted to be related to the interconnected processes of oxidative stress and inflammation. Over time, levels of naturally occurring antioxidant mechanisms decrease and contribute less towards counteracting the consequences of the brain's high oxygen usage. Studies show that generating oxidation and inflammation by radiation [1-3] or chemical agents [4, 5] can result in a pattern of changes in brain function and behavioral measures similar to those seen with advancing age. Furthermore, levels of inflammation have been associated with the amount of impairment found in cognitive and motor abilities

\footnotetext{
*Corresponding author: Barbara Shukitt-Hale, PhD, USDA-ARS, HNRCA at Tufts University, 711 Washington Street, Boston, MA 02111, USA. Tel.: +1 617556 3118; Fax: +1 617556 3299; E-mail: Barbara.ShukittHale@ ARS.USDA.GOV.

1878-5093/16/\$35.00 @ 2016 - IOS Press and the authors. All rights reserved

This article is published online with Open Access and distributed under the terms of the Creative Commons Attribution Non-Commercial License.
} 
[6-8]. As these and other studies indicate, animal models are helpful in identifying deficits associated with normal aging as well as factors that may protect against age-related alterations in function.

A number of berry fruits have proven to have health-promoting properties, most prominently antioxidant and anti-inflammatory effects [see $[9,10]$ for review]. Research has demonstrated beneficial effects on a range of age-related conditions including cardiovascular and neurodegenerative diseases, certain cancers, and arthritis [11-14]. Even in the absence of disease, some berries have demonstrated beneficial effects in aging, reducing changes in motor and cognitive performance $[15,16]$.

Berries are rich in vitamins, minerals, and phytochemicals and score high on several measures of antioxidant capacity $[17,18]$. Blueberries, strawberries, cranberries and blackberries are among the fruits found to be high in polyphenolic components and antioxidant activity and to also ameliorate deficits in motor and cognitive performance in old rats [19-22]. Measures of strength, balance, and agility, as well as learning and memory, improved following as little as two months of dietary supplementation compared to old animals fed a normal well-balanced diet.

Red raspberries are rich in macro and micro nutrients and have a unique profile of polyphenols, rating high in ellagitannins and anthocyanins [23, 24]. Whole fruit preparations as well as isolated components have demonstrated beneficial effects, in vitro and in vivo, including antimutagenicity, antiangiogenesis, and antioxidant capacity $[10,25,26]$. Despite these indicators of the bioactive potency of raspberries, their potential to moderate behavioral changes related to age has yet to be characterized.

In order to assess the effects of red raspberries on age-related impairments in motor and cognitive performance, 19-month-old Fischer 344 (F344) rats were fed a normal well-balanced diet with or without the addition of $2 \%$ red raspberry extract prepared from fresh fruit. After 7-10 weeks on the diet, learning and memory was assessed in the Morris water maze (MWM), and motor performance was measured using a standard battery of tests [27]. The concentration and duration of supplementation was based on effective doses found in previous studies [19-22]. Red raspberries are high in polyphenolic content and antioxidant capacity and therefore are expected to reduce brain-based behavioral deficits associated with normal aging.

\section{Materials and methods}

\subsection{Subjects}

Thirty male F344 rats were individually housed in stainless steel mesh suspended cages, provided food and water ad libitum, and maintained on a $12 \mathrm{hr}$ light/dark cycle. Following acclimatization to the facility, the 19-month-old rats were weight-matched and then randomly assigned to the raspberry or control diet group ( $n=15 /$ group). Rats were fed the diet for 11 weeks. The rats were tested at 21 months of age on a battery of motor tests during week 7 of the diet, and on the Morris water maze (MWM) during weeks 9-10 of the diet. Food intake and body weight were measured periodically. All rats were observed daily for clinical signs of disease. During the course of the study, three rats in the control group died or were euthanized due to excessive weight loss. All aspects of the treatment of the subjects were approved by the Animal Care and Use Committee of our Center in compliance with all applicable laws and regulations as well as principles expressed in the National Institutes of Health Guide for the Care and Use of Laboratory Animals.

\subsection{Diets}

Farm fresh red raspberries were delivered to the laboratory (D. Munson, Maberry \& Maberry Berry Assoc., Lynden, WA). Whole ripe fruit was added to water in a 2:1 weight to volume ratio and then homogenized in a blender for 2 minutes. The recovered homogenate was centrifuged at $13,000 \mathrm{~g}$ for $15 \mathrm{~min}$ at $4^{\circ} \mathrm{C}$. The supernatant was frozen, crushed, and placed in a freeze-drier for approximately 7 days for reduction to a dry powder. The 
freeze-dried extract was shipped to Harlan Teklad (Madison, WI) where it was combined with a nutritionally well-balanced control diet (a modification of the NIH-31 diet) [16]. The amount of corn in the control diet was adjusted to match the $2 \%(20 \mathrm{~g} / \mathrm{kg}$ diet, $2 \% \mathrm{w} / \mathrm{w})$ fruit extract added to the raspberry diet.

\subsection{Psychomotor testing}

During diet week 7, five different tests of psychomotor behavior previously determined to be affected by age [27] were administered to each of the thirty subjects in a randomized order. Each test was performed once, separated by a break between tasks. Full details of the behavioral testing procedures are reported elsewhere [27]. Briefly, the tests included: 1) Rod walking, which measures psychomotor coordination and the integrity of the vestibular system by requiring the animal to balance on a stationary, $26 \mathrm{~mm}$ diameter horizontal rod; 2) Wire suspension, which measures muscle strength and the prehensile reflex, an animal's ability to grasp a horizontal wire with its forepaws and to remain suspended; 3) Accelerating rotarod, which measures fine motor coordination, balance, and resistance to fatigue by measuring the amount of time that a rat can remain standing/walking on a rotating, slowly accelerating $60 \mathrm{~mm}$ diameter rod; 4) Plank walking, which measures balance and coordination by exposing the rats to three different size widths of horizontal planks ( $38 \mathrm{~mm}, 25 \mathrm{~mm}, 13 \mathrm{~mm}$ ); and 5) Inclined screen, which measures muscle tone, strength, stamina, and balance by placing the animal on a wire mesh screen that is tilted 60 degrees to the horizontal plane of the floor. The amount of time the animal stayed on the apparatus before falling to a cushioned surface was measured up to a maximum of $60 \mathrm{~s}$ (rod, wire, planks), $360 \mathrm{~s}$ (rotarod), or $600 \mathrm{~s}$ (inclined screen). Number of turns and distance traveled during plank walking were also recorded.

\subsection{Cognitive testing}

Cognitive testing was performed during diet weeks 9-10 with the working memory version of the Morris Water Maze (MWM) to test spatial learning and memory [27-29]. The MWM is an age-sensitive learning paradigm that requires subjects to use spatial learning to find a hidden platform $(10 \mathrm{~cm}$ in diameter) submerged $2 \mathrm{~cm}$ below the surface of the water in a circular pool of room temperature water $(134 \mathrm{~cm}$ in diameter $\times 50 \mathrm{~cm}$ in height), and to remember its location from the previous trial. Accurate navigation is rewarded with escape from the water onto the platform that the rat uses distal cues to effectively locate. Rats are strong swimmers and the water maze is the standard measure of learning and memory employed in studies where food deprivation and food rewards are not compatible with experimental goals.

MWM testing was performed daily for 4 consecutive days, with a morning and an afternoon session, two trials each session, with a 10 minute intertrial interval between the two trials. Each rat was allowed 120 sec to escape onto the platform; if the rat failed to escape within this time, it was guided to the platform. Once the rat reached the platform, it remained there for $15 \mathrm{sec}$ (Trial 1; reference memory or acquisition trial). The rat was returned to its home cage between trials ( $10 \mathrm{~min}$ ). Trial 2 (the working memory or retrieval trial) used the same platform location and start position as Trial 1 . The platform and start locations were varied between sessions to encourage the use of spatial strategies. Latency to find the platform was measured and performances were videotaped and analyzed with image tracking software (HVS Image, Hampton, England) to determine swim speed and distance traveled to the platform.

\subsection{Statistical analysis}

For each measure, between-subjects analysis of variance (ANOVA) models comparing the diet groups were performed using Systat (SPSS, Inc., Chicago, IL) to test for statistical significance at the $p<0.05$ level. Days or 
trials, when appropriate, were included in the model as a within-subjects variable. To analyze working memory, separate $t$-tests were conducted for each group between the mean Trial 1 and Trial 2 latencies.

\section{Results}

There were no differences in weight $(p>0.05$; average weight \pm SEM: control group $=428.71 \pm 5.69$; raspberry group $=426.24 \pm 4.03)$ or food intake $(p>0.05$; average food intake \pm SEM: control group $=21.60 \pm 0.35$; raspberry group $=21.90 \pm 0.59$ ) between the groups during the study.

The raspberry diet group performed significantly better than the control diet group on several psychomotor tasks. Raspberry-fed rats had longer latencies to fall off the stationary $\operatorname{rod}(\mathrm{F}(1,25)=3.95 ; p \leq 0.05 ; \mathrm{Fig}$. 1A), the medium plank $(\mathrm{F}(1,25)=4.00 ; p<0.05$, Fig. 1B) and the inclined screen $(\mathrm{F}(1,25)=4.82 ; p<0.05$; Fig. 1C). There was no effect of diet on the wire suspension and rotarod tasks $(p>0.05)$.

Cognitive performance as measured by latency to find the platform in the MWM was assessed by separate $t$-tests between the two trial latencies for each group, in order to see if diet significantly improved performance from Trial 1 to Trial 2 within sessions. The raspberry diet did not significantly affect any measure of learning and memory evaluated by this task. On days 3-4, performance depends more on memory than on learning. As shown in Fig. 2, there was no difference in either diet group between mean trial 2 and trial 1 performance on days 3-4 for time taken to find the platform $(p>0.05)$. Despite the significant effect of diet on several measures
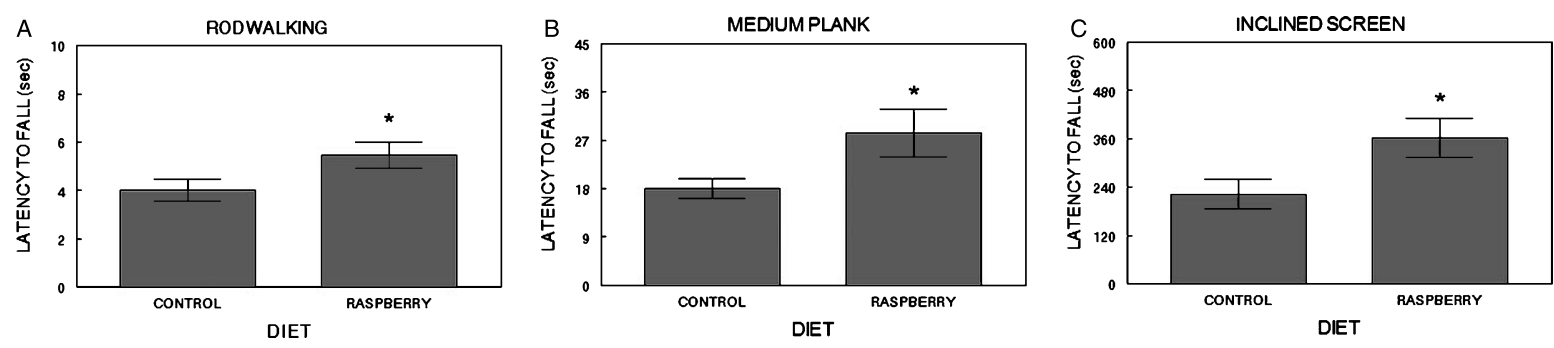

Fig. 1. Psychomotor performance: The $2 \%$ raspberry diet group had longer latencies to fall (mean \pm SEM: secs) on the rod walk (A), medium plank walk (B) and the inclined screen (C) compared to the control group $(*=p \leq 0.05$, one-way ANOVA).

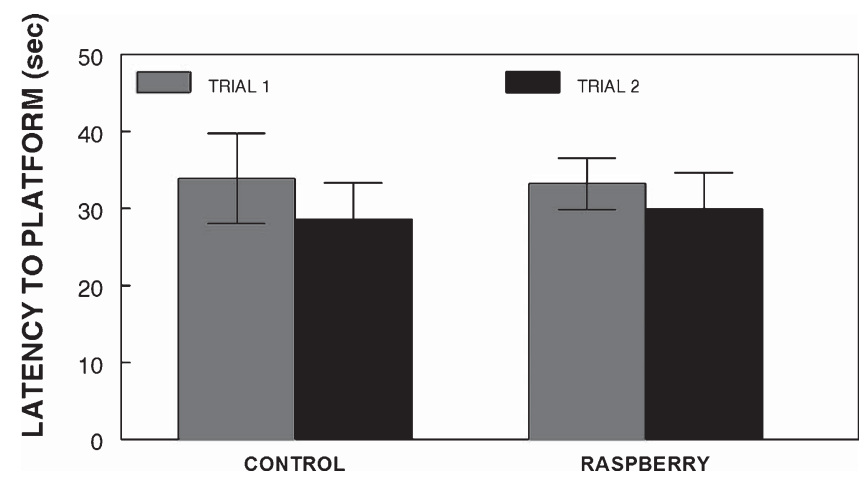

Fig. 2. Cognitive Performance: There was no difference in either diet group between mean trial 2 and trial 1 performance for latency (mean \pm SEM) to find the platform in the MWM, results for day 3 and 4 sessions depicted here $(p>0.05, t$-test). 
of motor function, there were no differences between the groups on distance traveled to the platform or swim speed in the water maze $(p>0.05)$.

\section{Discussion}

Ten weeks of red raspberry supplementation significantly improved motor skills of the old rats compared to old animals fed a standard well-balanced diet. In contrast, the raspberry diet did not significantly affect performance in the MWM, including measures of working and reference memory and swim speed. Specifically, old rats fed the $2 \%$ raspberry diet had better performance on the rod and plank walks, which measure psychomotor coordination and balance, and on the inclined screen, which measures muscle tone, strength, stamina, and balance.

That a diet supplemented with a polyphenol-rich food significantly improved markers of motor function without concomitant enhancement of learning and/or memory is unique within our model. A number of supplements have decreased age-related deficits in both motor and cognitive performance, e.g., blueberries, strawberries, and grape juice, or solely affected measures of learning and memory as assessed in the MWM, e.g., black currants, plum juice, and spinach [19-22, 30-32]. Raspberries, when added to a well-balanced diet, are distinguished by their ability to impact psychomotor performance alone.

The dose and relative bioavailability of antioxidant and anti-inflammatory components may underlie the profile of behavioral improvements resulting from ten weeks of red raspberry supplementation. The concentration of anthocyanins found in the brain following a blueberry-supplemented diet has been shown to positively correlate with the level of improvement in cognitive performance in old rats [33]. In another series of experiments, rats received either a $10 \%$ or a $50 \%$ grape juice supplement or an energy-matched placebo from age 19 to 21 months [30]. Measures of cognitive and psychomotor performance similar to those used in the current study demonstrated a clear dose-dependent effect. MWM performance improved with the $10 \%$ supplement, but not a $50 \%$ supplement, while motor function was improved only in the $50 \%$ grape juice group. It is possible that feeding animals a higher, or perhaps lower, concentration of raspberry extract might extend the anti-aging effects to include improved learning and memory.

Although red raspberries have been shown to have antioxidant and anti-inflammatory effects in a number of cell culture models, in vivo studies are relatively rare [34, 35]. One animal study did find a dose-dependent reduction of signs of disease including decreased cartilage inflammation following thirty days of administration of a raspberry extract in a rat model of arthritis [13]. Experimenters noted that articular cartilage necessary for load-bearing strength was protected against inflammation and damage by the high dose but not the low dose of extract. Although the doses and extract preparation varied substantially from the current study, the results do confirm that short-term berry supplementation can have peripheral effects benefiting motor capabilities.

Red raspberries have a unique profile of bioactive components and are unusual compared to many other conventional berries. Along with being rich in polyphenols and other antioxidants, including anthocyanins and Vitamin C, they are distinguished by containing large amounts of ellagitannins which account for over half of their antioxidant capacity [17]. Current measures of the pharmacokinetics and bioavailability of ellagitannins indicate that they are poorly absorbed in humans and rats, suggesting that they may possibly exert the majority of their effects within the gastrointestinal tract [17, 23, 36]. Diverse arrays of dietary components have been tested as agents for moderating inflammation and oxidative stress. Yet, evidence suggests that in vivo whole foods that contain a multiplicity of potentially synergistic bioactive compounds may be most efficacious [37, 38]. Further research is needed to determine the mechanisms by which dietary supplementation with red raspberries prevented age-related psychomotor decline.

Nutritional interventions, including dietary supplementation with polyphenol-rich berries, show promise for decreasing behavioral deficits associated with aging. In this study, red raspberries were found to significantly improve measures of strength, balance and coordination. Given that falls are the number one health hazard for otherwise healthy senior citizens, these results may have important implications for increasing healthy aging. 


\section{Acknowledgments}

This study was supported in part by USDA Intramural funds and the Washington Red Raspberry Commission.

\section{References}

[1] Joseph JA, Shukitt-Hale B, McEwen J, Rabin BM. CNS-induced deficits of heavy particle irradiation in space: The aging connection. Adv Space Res. 2000;25(10):2057-64.

[2] Joseph JA, Erat S, Rabin BM. CNS effects of heavy particle irradiation in space: Behavioral implications. Adv Space Res. 1998;22(2):209-16.

[3] Shukitt-Hale B, Casadesus G, Carey AN, Rabin BM, Joseph JA. Exposure to 56Fe irradiation accelerates normal brain aging and produces deficits in spatial learning and memory. Advances in Space Research. 2007;39(6):1087-92.

[4] Shukitt-Hale B, Lau FC, Carey AN, Galli RL, Spangler EL, Ingram DK, et al. Blueberry polyphenols attenuate kainic acid-induced decrements in cognition and alter inflammatory gene expression in rat hippocampus. Nutr Neurosci. 2008;11(4):172-82.

[5] Duffy KB, Spangler EL, Devan BD, Guo Z, Bowker JL, Janas AM, et al. A blueberry-enriched diet provides cellular protection against oxidative stress and reduces a kainate-induced learning impairment in rats. Neurobiol Aging. 2008;29(11):1680-9.

[6] Hauss-Wegrzyniak B, Vannucchi MG, Wenk GL. Behavioral and ultrastructural changes induced by chronic neuroinflammation in young rats. Brain Res. 2000;859(1):157-66.

[7] Hauss-Wegrzyniak B, Vraniak P, Wenk, GL. The effects of a novel NSAID on chronic neuroinflammation are age dependent. Neurobiol Aging. 1999;20(3):305-13.

[8] Shukitt-Hale B. The effects of aging and oxidative stress on psychomotor and cognitive behavior. Age (Omaha). 1999;22(1):9-17.

[9] Stevenson DE, Hurst RD. Polyphenolic phytochemicals-just antioxidants or much more? Cell Mol Life Sci. 2007;64(22):2900-16.

[10] Seeram NP. Berries. In: Heber D, editor. Nutritional oncology. 2nd ed. Amsterdam; Boston: Elsevier-Academic Press; 2006, p. xxiv, $822 \mathrm{p}$.

[11] Seeram NP, Adams LS, Zhang Y, Lee R, Sand D, Scheuller HS, et al. Blackberry, black raspberry, blueberry, cranberry, red raspberry, and strawberry extracts inhibit growth and stimulate apoptosis of human cancer cells in vitro. J Agric Food Chem. 2006;54(25):9329-39.

[12] Zafra-Stone S, Yasmin T, Bagchi M, Chatterjee A, Vinson JA, Bagchi D. Berry anthocyanins as novel antioxidants in human health and disease prevention. Mol Nutr Food Res. 2007;51(6):675-83.

[13] Jean-Gilles D, Li L, Ma H, Yuan T, Chichester CO, 3rd, Seeram NP. Anti-inflammatory effects of polyphenolic-enriched red raspberry extract in an antigen-induced arthritis rat model. J Agric Food Chem. 2012;60(23):5755-62.

[14] Singh M, Arseneault M, Sanderson T, Murthy V, Ramassamy C. Challenges for research on polyphenols from foods in Alzheimer's disease: Bioavailability, metabolism, and cellular and molecular mechanisms. J Agric Food Chem. 2008;56(13):4855-73.

[15] Joseph JA, Shukitt-Hale B, Willis LM. Grape juice, berries, and walnuts affect brain aging and behavior. J Nutr. 2009;139(9):1813S-7S.

[16] Youdim KA, Shukitt-Hale B, Martin A, Wang H, Denisova N, Bickford PC, et al. Short-term dietary supplementation of blueberry polyphenolics: Beneficial effects on aging brain performance and peripheral tissue function. Nutr Neurosci. 2000;3: 383-97.

[17] Borges G, Degeneve A, Mullen W, Crozier A. Identification of flavonoid and phenolic antioxidants in black currants, blueberries, raspberries, red currants, and cranberries. J Agric Food Chem. 2010;58(7):3901-9.

[18] Wolfe KL, Kang X, He X, Dong M, Zhang Q, Liu RH. Cellular antioxidant activity of common fruits. J Agric Food Chem. 2008;56(18):8418-26.

[19] Shukitt-Hale B, Cheng V, Joseph JA. Effects of blackberries on motor and cognitive function in aged rats. Nutr Neurosci. 2009;12(3):135-40.

[20] Shukitt-Hale B, Galli RL, Meterko V, Carey A, Bielinski DF, McGhie T, et al. Dietary supplementation with fruit polyphenolics ameliorates age-related deficits in behavior and neuronal markers of inflammation and oxidative stress. Age (Dordr). 2005;27(1):49-57.

[21] Shukitt-Hale B, Bielinski DF, Lau FC, Willis LM, Carey AN, Joseph JA. The beneficial effects of berries on cognition, motor behaviour and neuronal function in ageing. Br J Nutr. 2015;114(10):1542-9.

[22] Joseph JA, Shukitt-Hale B, Denisova NA, Bielinski D, Martin A, McEwen JJ, et al. Reversals of age-related declines in neuronal signal transduction, cognitive, and motor behavioral deficits with blueberry, spinach, or strawberry dietary supplementation. J Neurosci. 1999;19(18):8114-21.

[23] Borges G, Roowi S, Rouanet JM, Duthie GG, Lean ME, Crozier A. The bioavailability of raspberry anthocyanins and ellagitannins in rats. Mol Nutr Food Res. 2007;51(6):714-25.

[24] Lugasi A, Hóvári J, Kádár G, Dénes F. Phenolics in raspberry, blackberry and currant cultivars grown in Hungary. Acta Alimentaria. 2011;40(1):52-64. 
[25] Bradish CM, Perkins-Veazie P, Fernandez GE, Xie G, Jia W. Comparison of flavonoid composition of red raspberries (Rubus idaeus L.) grown in the southern United States. J Agric Food Chem. 2012;60(23):5779-86.

[26] Beekwilder J, Hall RD, de Vos CH. Identification and dietary relevance of antioxidants from raspberry. Biofactors. 2005;23(4): 197-205.

[27] Shukitt-Hale B, Mouzakis G, Joseph JA. Psychomotor and spatial memory performance in aging male Fischer 344 rats. Exp Gerontol. 1998;33(6):615-24.

[28] Brandeis R, Brandys Y, Yehuda S. The use of the Morris water maze in the study of memory and learning. Intern J Neurosci. 1989;48:29-69.

[29] Morris R. Development of a water-maze procedure for studying spatial learning in the rat. J Neurosci Meth. 1984;11:47-60.

[30] Shukitt-Hale B, Carey A, Simon L, Mark DA, Joseph JA. Effects of Concord grape juice on cognitive and motor deficits in aging. Nutrition. 2006;22(3):295-302.

[31] Shukitt-Hale B, Kalt W, Carey AN, Vinqvist-Tymchuk M, McDonald J, Joseph JA. Plum juice, but not dried plum powder, is effective in mitigating cognitive deficits in aged rats. Nutrition. 2009;25(5):567-73.

[32] Willis LM, Shukitt-Hale B, Cheng V, Joseph JA. Dose-dependent effects of walnuts on motor and cognitive function in aged rats. Br J Nutr. 2009;101(8):1140-4.

[33] Andres-Lacueva C, Shukitt-Hale B, Galli RL, Jauregui O, Lamuela-Raventos RM, Joseph JA. Anthocyanins in aged blueberry-fed rats are found centrally and may enhance memory. Nutr Neurosci. 2005;8(2):111-20.

[34] Rao AV, Snyder DM. Raspberries and human health: A review. J Agric Food Chem. 2010;58(7):3871-83.

[35] Seeram NP. Berry fruits for cancer prevention: Current status and future prospects. J Agric Food Chem. 2008;56(3):630-5.

[36] Gonzalez-Barrio R, Borges G, Mullen W, Crozier A. Bioavailability of anthocyanins and ellagitannins following consumption of raspberries by healthy humans and subjects with an ileostomy. J Agric Food Chem. 2010;58(7):3933-9.

[37] Manganaris GA, Goulas V, Vicente AR, Terry LA. Berry antioxidants: Small fruits providing large benefits. J Sci Food Agric. 2014;94(5): 825-33.

[38] Nile SH, Park SW. Edible berries: Bioactive components and their effect on human health. Nutrition. 2014;30(2):134-44. 